\title{
Model-Based Tabu Search Algorithm for Free-Space Optical Communication with a Novel Parallel Wavefront Comection System
}

\author{
Zhaokun $\mathrm{Li}^{1}$, Xiaohui Zhao ${ }^{1 *}$, Jingtai $\mathrm{Cao}^{1,2}$, and Wei Liu ${ }^{1}$ \\ ${ }^{1}$ College of Communication Engineering, Jilin University, 5372 Nanhu Road, Changchun 130012, \\ P. R. China \\ ${ }^{2}$ Changchun Institute of Optics, Fine Mechanics and Physics, Chinese Academy of Sciences, \\ 3888 Nanhu Road, Changchun 130033, P. R. China
}

(Received July 25, 2014 : revised December 30, 2014 : accepted December 30, 2014)

\begin{abstract}
In this study, a novel parallel wavefront correction system architecture is proposed, and a model-based tabu search (MBTS) algorithm is introduced for this new system to compensate wavefront aberration caused by atmospheric turbulence in a free-space optical (FSO) communication system. The algorithm flowchart is presented, and a simple hypothetical design for the parallel correction system with multiple adaptive optical (AO) subsystems is given. The simulated performance of MBTS for an AO-FSO system is analyzed. The results indicate that the proposed algorithm offers better performance in wavefront aberration compensation, coupling efficiency, and convergence speed than a stochastic parallel gradient descent (SPGD) algorithm.
\end{abstract}

Keywords: Parallel correcting system architecture, Model based tabu search algorithm, Free-space optical communications, Atmospheric turbulence

OCIS codes : (110.1080) Active or adaptive optics; (200.2605) Free-space optical communication; (010.3310) Laser beam transmission; (060.4510) Optical communications

\section{INTRODUCTION}

Recently free-space optical (FSO) communication has attracted more and more attention as it becomes widely used among the telecommunication community for both ground- and space-based wireless links and "last-mile" applications [1], due to its unregulated spectrum, high potential bandwidth, relatively low power requirement, low bit error rate, and ease of redeployment. However, phase disturbances from atmospheric turbulence along propagation paths, manifesting as intensity fluctuation (scintillation), beam wandering, and beam broadening at the receiver all lead to significant decrease of coupling efficiency [2], which seriously influences the stability and reliability of FSO communication systems [3].

An adaptive optical (AO) system is an effective method to improve laser-beam quality by correcting any wavefront aberration, and has already allowed great achievements [4-9]. Generally, in a conventional AO system a Shack-Hartmann wavefront sensor (S-H sensor) [10] measures the optical phase deviations of an incoming wavefront, and a deformable mirror
(DM) is used to compensate for the phase distortion. Then the DM generates a wavefront phase to compensate for the phase aberration based on phase-conjugation theory [11, 12].

Under strong scintillation, sensorless AO systems are proposed to compensate for the wavefront aberration. Some very effective blind control optimized algorithms, such as stochastic parallel gradient descent (SPGD) and simulated annealing (SA) have been proposed to improve the performance of the FSO system [13]. In this study, a new method called the model-based tabu search (MBTS) algorithm is proposed to offer better performance in atmospheric turbulence compensation. Tabu search (TS) is a meta-heuristic random-search algorithm [14] that starts from an initial solution and selects some specified directions for probing. To avoid being trapped in a local minimum, TS uses a flexible method to record the process of optimization and selection to guide the next search step, while a Zernike model-based (MB) method in an $\mathrm{AO}$ system can decrease the dimension of the search space, relieve calculation complexity and accelerate convergence

\footnotetext{
*Corresponding author:xhzhao@jlu.edu.cn

Color versions of one or more of the figures in this paper are available online.
} 
speed. Model-based tabu search (MBTS), the combination of MB and TS approaches, will benefit from these two methods and perform better in an FSO system, since it can effectively improve coupling efficiency at the receiver and reduce energy loss in the laser propagation path. Most important is that MBTS uses very few iterations, far less than does SPGD (which usually needs hundreds of iterations). Moreover, to further improve the performance of the MBTS algorithm, a novel parallel correcting system is proposed. The newly invented system can increase the speed of correction of the wavefront aberration by simply combining several uniform $\mathrm{AO}$ subsystems together, each subsystem correcting certain orders of aberration by MBTS. The working principle of this new system is explained further.

This paper is organized as follows: Section 2 provides models of an FSO communication system, sensorless AO system, and DM. Section 3 gives an analysis of MBTS algorithm and its working principles in the FSO communication system, as well as a simple and novel hypothetical design for a parallel correcting system with multiple AO subsystems. In Section 4, some simulations are carried out to show the performance of the proposed MBTS in a sensorless AO-FSO system, in comparison with SPGD algorithm. Finally, some conclusions from this study are given in Section 5 .

\section{METHODS}

\subsection{FSO Communication System with Model - Based Tabu Search Algorithm}

A laser communication system in atmospheric turbulence works poorly, because an aberrated wavefront leads to energy loss in receiver. In an ideal case when the system does not introduce any aberration to the laser signal, all fluorescence is emitted from a point, so that the laser is well coupled to a single mode fiber (see Fig. 1 (a)). When atmospheric turbulence is present, the intensity in the focal plane depends on the wavefront of incident light, which spreads widely over the focal plane of lens due to the wavefront aberration; thus the coupling efficiency at the receiver will degrade, and the bit error rate will increase (Fig. 1 (b)) [15].

The simple schematic diagram of an FSO experimental setup is illustrated in Fig. 2. A laser from a distant source passes through an atmospheric disturbances box, in which the wavefront is disturbed by the atmospheric turbulence device. In a conventional AO system, when the laser enters it is reflected by a deformable mirror (DM) and directed to a wavefront sensor (WFS). The measured signal is sent to a controller to reconstruct the wavefront from the WFS measurement, and to compare the reconstructed wavefront to a reference plane wavefront for computation of the control

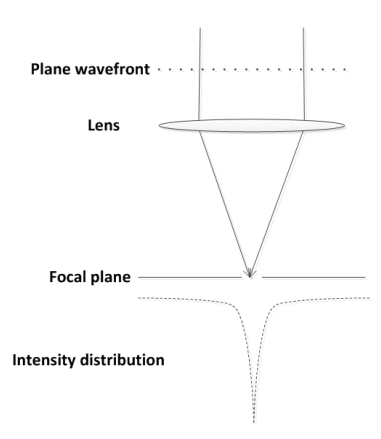

(a)

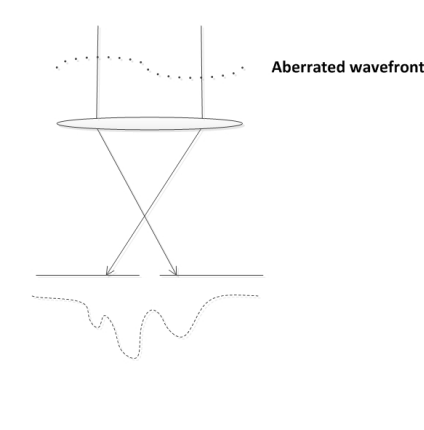

(b)
FIG. 1. Effect of aberrant wavefront on intensity: (a) laser with plane wavefront, (b) laser with aberrant wavefront.

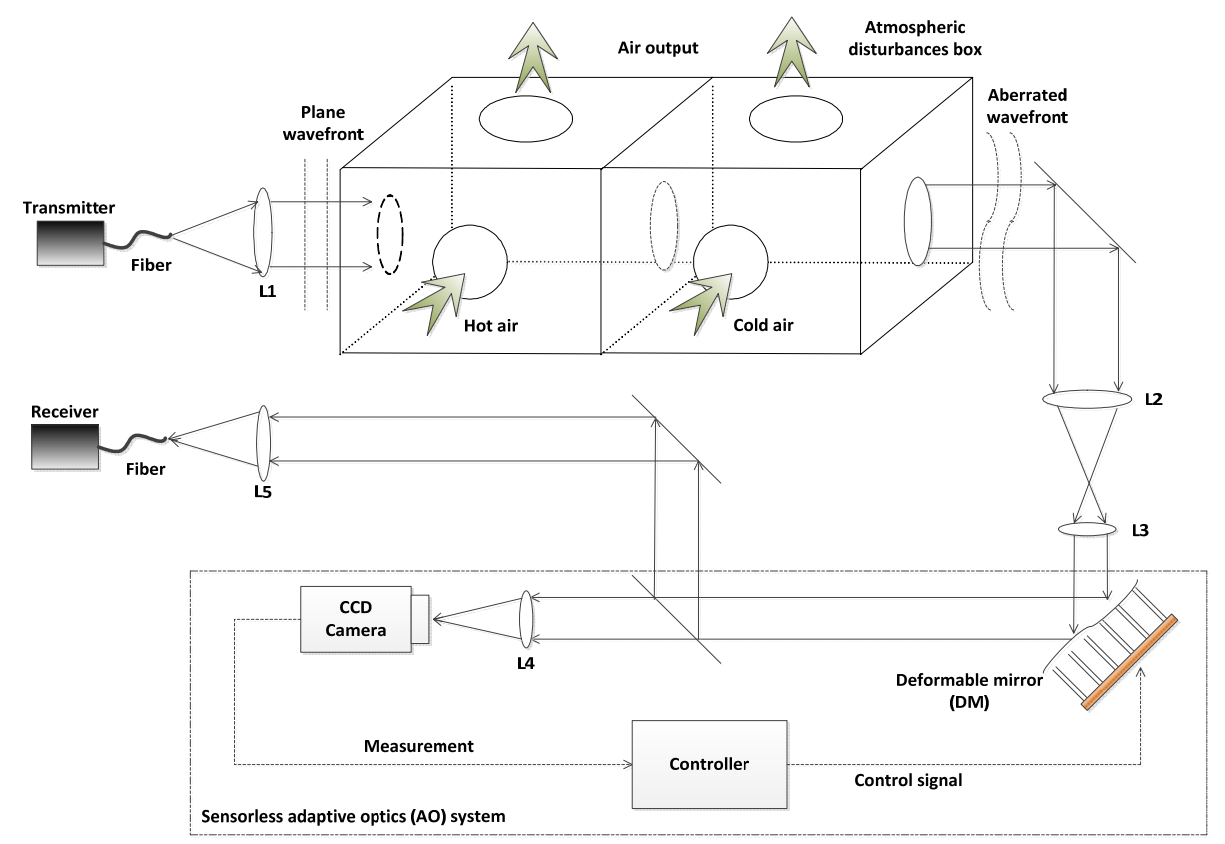

FIG. 2. FSO system with sensorless AO. 
signal for the DM. Consequently, the DM deforms its surface to counteract the wavefront aberration. In this way the laser wavefront is compensated, and thus the coupling efficiency in the communication receiver increases. However, in a recently proposed sensorless AO system, a CCD camera replaces the WFS. This camera measures the intensity at the focus plane to obtain a performance metric and generate a good control signal vector. In addition, a good algorithm should also provide a better solution in fewer iterations for a real-time system in practice.

Our goal is to find a control signal so that the DM can generate the best surface deformation to compensate for the wavefront aberration, so that in turn more laser energy is coupled into the single mode fiber.

\subsection{Wavefront Compensation Device}

In general, a DM with more actuators could generate a more precise correcting phase to compensate for lower-order but higher-value aberration. However, a DM with too many actuators and too large a receiving aperture is not useful in laser transmission, because it requires reshaping of the laser beam to a large diameter to match the receiving aperture. In our analysis, a DM with 32 actuators is a suitable selection that generates an acceptable result and has no excessive requirement for beam reshaping. The normalized layout of the 32-element DM actuators is shown in Fig. 3.

We approximate the DM influence function by a Gaussian mdel as follows:

$$
S_{j}(x, y)=\exp \left\{\ln \omega\left[\frac{1}{d} \sqrt{\left(x-x_{j}\right)^{2}+\left(y-y_{j}\right)^{2}}\right]^{\alpha}\right\}
$$

where $\omega$ is the coupling coefficient determined by the sizes of the electrode actuators and the DM, $\left(x_{j}, y_{j}\right)$ are the coordinates of the center of the $j$ th actuator, $d$ is the interval between the adjacent actuators, and $\alpha$ is the Gaussian index. The phase compensation $\phi(x, y)$ generated by the deformable mirror is given by

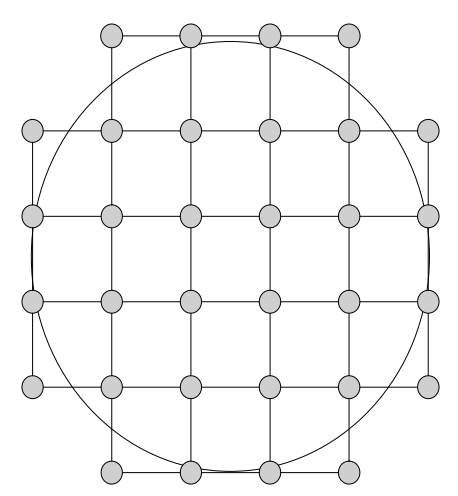

FIG. 3. Layout of actuators (filled circles) for a 32-element deformable mirror. The layout of the actuators is a square arrangement.

$$
\phi(x, y)=\sum_{j=1}^{32} v_{j} S_{j}(x, y)
$$

where $v_{j}$ is the $j$ th voltage of the actuators, and $(x, y)$ is the location of each point on the DM. The numerical relationship between the aberration generated by the DM and the voltages $v_{j}$ applied to the actuators is linear.

\subsection{Model-based Compensation in Adaptive Optics}

From the conventional point of view, we often pay lot of attention to the control voltages applied to the actuators, but problems occur due to a solution space of high dimension, which can result in slow convergence, large computational burden, and unsatisfied convergence value of the control algorithm, since increasing the number of actuators brings a geometric increase in the number of calculations of the control solution. This is important for real-time performance of the system. A new, alternative approach is to use a Zernike model to fit induced wavefront aberration and to indirectly get appropriate voltages, instead of directly using a DM model. No matter how many actuators exist, we only need to obtain the linear relationship between the control voltages and Zernike coefficients, so that the number of calculations will decrease, which is better for an FSO system under high-speed workload.

We assume the wavefront aberration $\phi$ and correcting aberration $\varphi$ can be represented by a series of $M$ and $N$ Zernike functions, respectively. In most cases, $M>N$. The definitions of $\phi$ and $\varphi$ are

$$
\begin{aligned}
& \phi(x, y)=\sum_{i=1}^{M} w_{i} \cdot Z_{i}(x, y) \\
& \varphi(x, y)=\sum_{i=1}^{N} u_{i} \cdot Z_{i}(x, y)
\end{aligned}
$$

In other words, the aberration of the incident wavefront $\varphi$ can be denoted by the vector $W$, whose elements are the coefficients $w_{i}$. Similarly, the correcting aberration $\varphi$ and the residual aberration $\mathfrak{R}$ can be represented by the vectors $\mathbf{U}$ and $W$ with elements $u_{i}$ and $w_{i}$ respectively and $\mathfrak{R}_{i}=w_{i}-u_{i}$ [16]. To get the best $\mathbf{U}$ through the minimization of $\operatorname{var}(\mathfrak{R})$ is our goal for the sensorless AO system.

In practice, the correcting aberration $\varphi$ is excited by the actuator voltages from the DM, which is approximated by Eq. (2). The voltage $v_{j}$ applied to the $j$ th actuator determines the correcting aberration $\varphi$. Ideally, the wavefront aberration fitting by the Zernike coefficients (Eq. (4)) and the phase aberration generated by the DM (Eq. (2)) should be identical, even if this cannot be completely realized. From Eq. (2) and (4) we obtain

$\mathbf{S V} \approx \mathbf{Z U}$ 
where

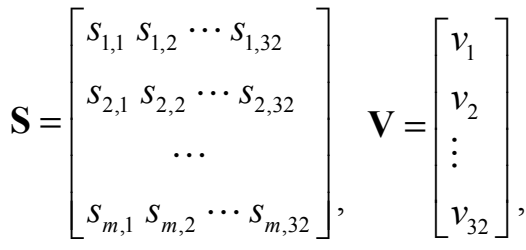

$$
\begin{aligned}
& \mathbf{Z}=\left[\begin{array}{cccc}
z_{1,1} & z_{1,2} & \cdots & z_{1, N} \\
z_{2,1} & z_{2,2} & \cdots & z_{2, N} \\
\cdots & & \\
z_{m, 1} & z_{m, 2} & \cdots & z_{m, N}
\end{array}\right], \quad \mathbf{U}=\left[\begin{array}{l}
u_{1} \\
u_{2} \\
\vdots \\
u_{N}
\end{array}\right]
\end{aligned}
$$

and $S_{i, k}$ is the $k$ th effect of the voltage on the $i$ th sampled value, and $Z_{i, k}$ is the $k$ th Zernike coefficient for the $i$ th sampled value. Here we have $m$ sampled values. If $\mathbf{U}$ is obtained, the vector $\mathbf{A}$ is

$$
\mathbf{V} \approx \mathbf{S}^{\dagger} \mathbf{Z U}=\mathbf{B U}
$$

where $\mathbf{S}^{\dagger}$ is the pseudo-inverse of $\mathbf{S}$. Obviously it can be calculated offline and is simplified; what we need to do is simply find the best $\mathbf{U}=\left(u_{1}{ }^{*}, u_{2}{ }^{*}, \cdots u_{N}{ }^{*}\right)$.

\subsection{Parallel Wavefront Correcting System}

The sensorless AO system described above cannot take the speed of the proposed algorithm to the extreme, so here we put forward a new system architecture that combines many uniform $\mathrm{AO}$ subsystems, to increase the processing rate.

We outline a simple conception of this equipment with four AO systems based on the algorithm proposed in this paper, as examplified in Fig. 4. The incident light is divided into some number of parts by beam splitters. Each part of light passes through a sensorless $\mathrm{AO}$ subsystem and is corrected by the MBTS algorithm: AO subsystem I gets the best solution $\left(u_{1}{ }^{*}, u_{2}{ }^{*}\right), \mathrm{AO}$ subsystem II gets the best solution $\left(u_{3}{ }^{*}, u_{4}{ }^{*}\right)$, and so on. A master control system obtains the best voltage solutions based on the best Zernike mode fitting solutions from $u_{1}{ }^{*}$ to $u_{7}{ }^{*}$ and Eq. (6) (mentioned in Section 3.1). In our opinion, the light should not be divided into too many parts, to avoid difficulty in measurement due to weak light intensity at the CCD camera. In our analysis, each AO subsystem deals with two orders, and the optimum number of parallel AO systems is $\operatorname{ceil}(\mathrm{N} / 2)$, which is the smallest integer bigger than $\mathrm{N} / 2$ (where $\mathrm{N}$ is the order of aberration to be compensated); it is sufficient and easily selected, based on the specific circumstances.

\subsection{Tabu Search Algorithm}

The tabu search (TS) algorithm is an effective way to find an optimal or nearly optimal global solution, which can guide the search process to avoid a local optimum and find the global optimum. The TS algorithm begins with an initial solution $S$, finds a set of neighborhoods $N(S)$, selects a new solution $S^{*}$ from $N(S)$ according to some rules, transforms from $S$ to $S^{*}$, and repeats this process until the termination condition is met. To avoid meaningless cycling and becoming trapped in a local optimal solution, a tabu list of length $L$ is introduced in this algorithm. The elements in the list are the solutions that have been selected from $N(S)$. In other words, they are the latest $L$ statuses to have been selected; in future processes, we cannot select these solutions for a certain number of iterations.

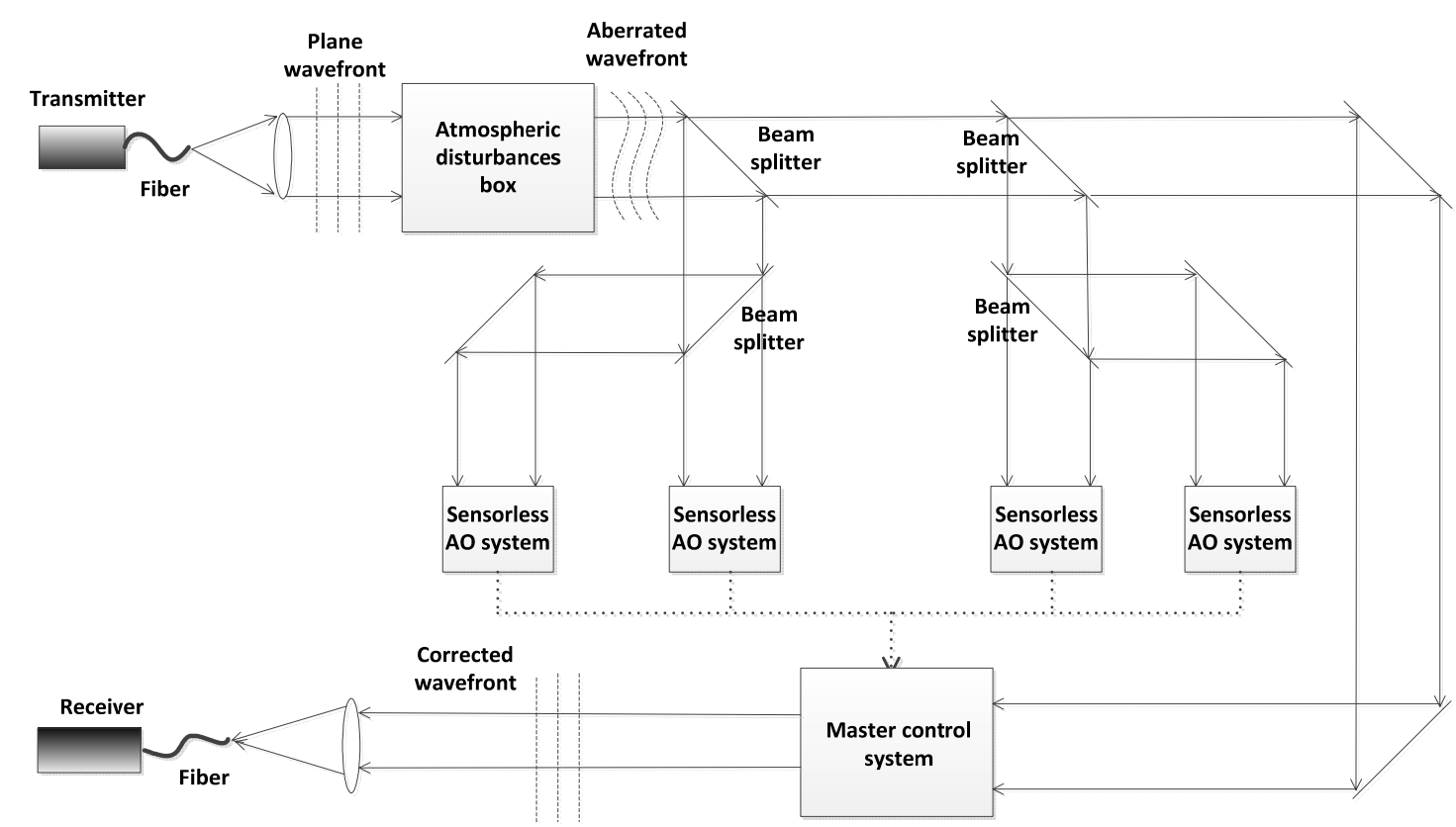

FIG. 4. Parallel processing system with multiple sensorless AO subsystems. 
In general, the TS algorithm selects a new solution according to a rule such as this: If the new $S^{*}$ is in the tabu list, whether it should be selected or not is based on its corresponding fitness. If the fitness is better than the current best value, then it can be selected directly; otherwise it is disregarded, and $S^{*}$ is replaced by the second-best solution within $N(S)$. Hence we find whether it is in the tabu list or not, and this cycle repeats.

The detailed process is described as follows:

(1) Select an initial solution. A good initial solution is very import to finding a global optimal solution. Here it is the Zernike coefficients that generate the correcting aberration $\varphi$. The proper approach is to know the environmental conditions, record the phase aberration for as long as possible, and use the expectation value as the initial condition. In the simulation, we take zero as a suitable starting point, assuming that the processing rate is fast compared to the DM's frame rate.

(2) Find an appropriate $N(S)$. To reduce the number of calculations, we decompose the solution into different groups, i.e. we take coefficients $v_{1}$ and $v_{2}$ as a group $\left(v_{1}, v_{2}\right)$ and further groups $\left(v_{3}, v_{4}\right),\left(v_{5}, v_{6}\right), \ldots$, respectively, such that the search occurs in a twodimensional space (see Fig. 5). Here we choose $N(S)$ as a group of eight points adjacent to $S$ (shown in Fig. 6). Each point in $N(S)$ is a candidate solution

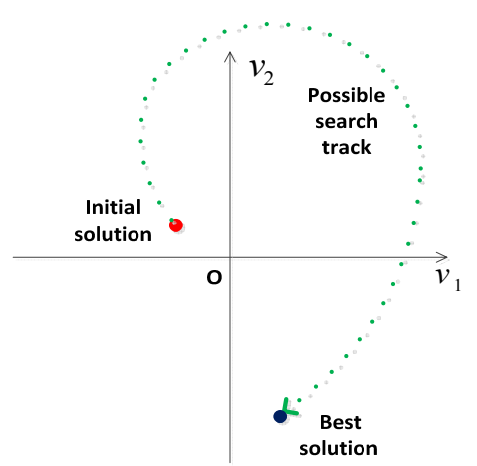

FIG. 5. Two-dimensional search space.

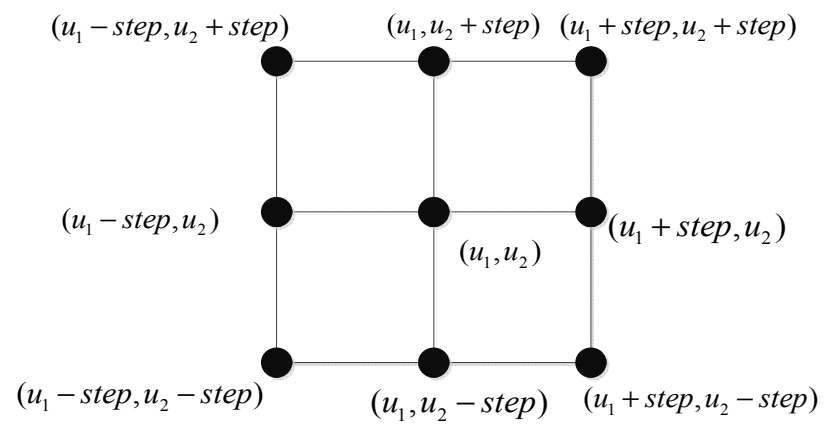

FIG. 6. The eight adjacent groups for $N(S)$.

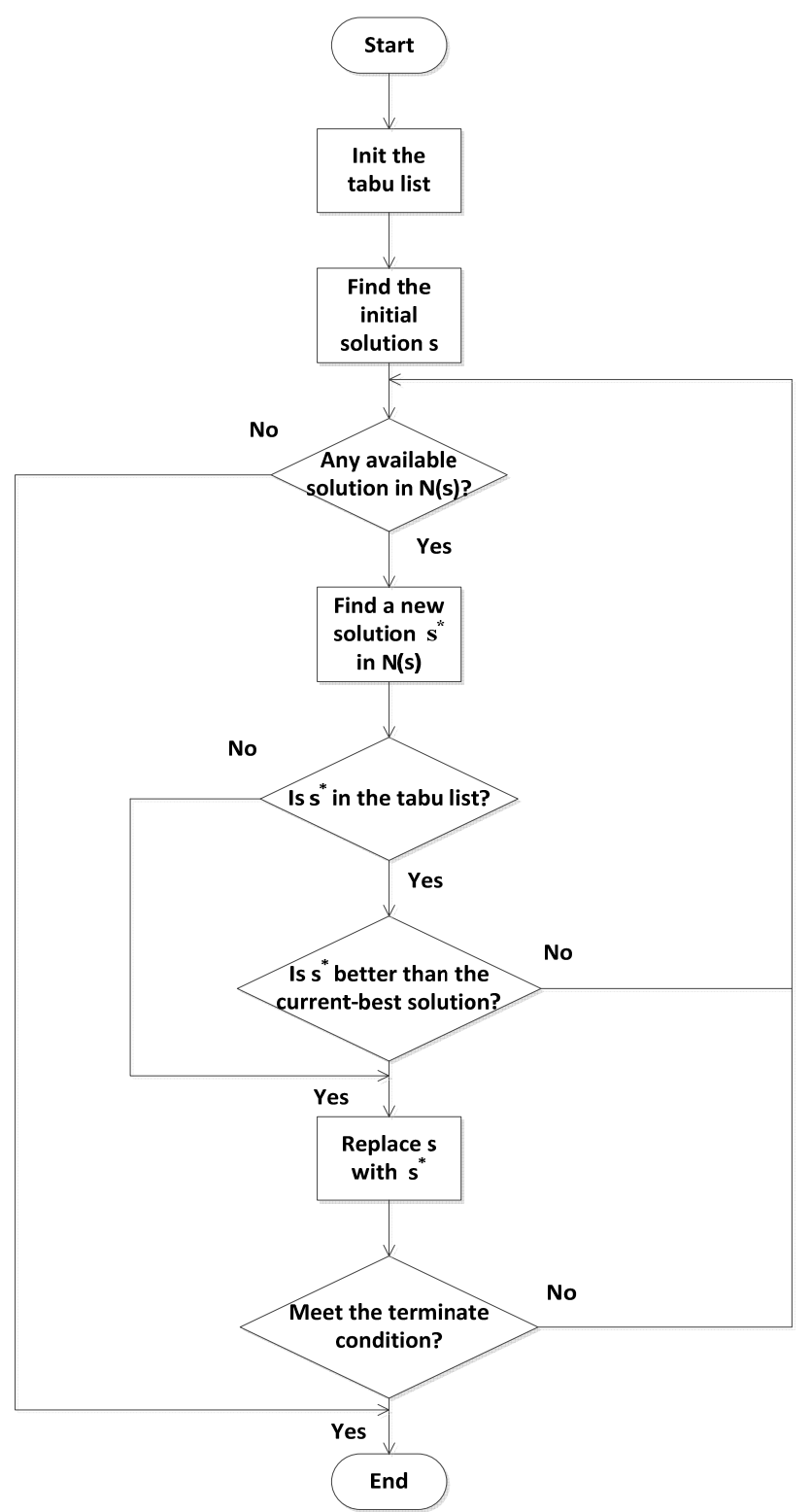

FIG. 7. Flow chart of the TS algorithm.

that may be selected. During the search, the fitness is the variance of the residual wavefront phase $\operatorname{var}(\mathfrak{R})$.

(3) Select a new solution $S^{*}$ from $N(S)$ by the abovementioned rules, then append it to the tabu list (delete the first record if the list has no place for the new solution $S^{*}$, hence the deleted record could be selected again in a future search), and replace $S$ with $S^{*}$.

(4) If the termination condition is satisfied, we have obtained the best solution; otherwise, go to step (2) and loop.

The flow chart is shown as Fig. 7.

\subsection{Performance Metric of our FSO System}

The goal of an adaptive optical system is to minimize 
residual phase aberrations after an incoming wave passes the deformable mirror. This corresponds to a maximization of the Strehl ratio (ST). In this paper, we use ST as the system performance metric $J$ [17],

$$
J \equiv \mathrm{ST} \propto\left|A\left(r_{0}\right)\right|^{2}
$$

where $A(r)$ is the (complex) optical field in the focal plane, and $r_{0}$ is the desired on-axis location of the center of the fiber end within this plane. In this study, a CCD camera is used to measure the optical power emerging from the hole; the measured quantity is thus proportional to the integral of the intensity within the pinhole with radius $a$, and we have an experimental performance metric [18-20]

$$
J \propto \iint_{\left|r-r_{0}\right|<a}\left|A\left(r_{0}\right)\right|^{2} d^{2} r
$$

For direct optimization of the power coupled into a fiber, the system performance metric $J$ is proportional to the absolute coupling efficiency. In case of a single-mode fiber, the theoretical value is proportional to the overlap integral of the optical field $A(r)$ and the mode profile $M_{0}(r)$ in the focal plane; thus the performance metric $J$ is given by

$$
J \propto \frac{\left|\iint A_{f}(r) M_{0}^{*}(r) d^{2} r\right|^{2}}{\iint A_{f}(r) A_{f}^{*}(r) d^{2} r \times \iint M_{0}(r) M_{0}^{*}(r) d^{2} r}
$$

Assume that the wavefront phase aberration satisfies a Gaussian distribution, ST can be estimated by variance $\mathrm{RMS}^{2}$ as follow

$$
\mathrm{ST} \propto \exp \left(-\mathrm{RMS}^{2}\right)
$$

With increasing coupling efficiency, more energy is coupled into the single-mode fiber. When $\mathrm{RMS}^{2}$ is close to 0 , we can get a simpler formula:

$$
\mathrm{ST} \propto 1-\mathrm{RMS}^{2}
$$

In practice, the pixel size of the CCD camera approximately equals the fiber diameter, so ST is [18]

$$
\mathrm{ST}=\frac{|\max [A(i)]|^{2}}{\left|\sum_{i=1}^{P}[A(i)]^{2}\right|}
$$

where $A(i)$ is the gray value of the $i$ th pixel, and $P$ is the number of pixels.

\section{RESULTS}

Based on the previous analysis, 12 Zernike modes and the corresponding correcting aberration are given in Fig. 8.

Define the fitting error as fitting capability for the wavefront aberration of DM [18]

$$
\mathrm{E}_{f e}=\frac{\mathrm{RMS}_{R}}{\mathrm{RMS}_{C}}
$$

where $\mathrm{RMS}_{C}$ represents the RMS value of the wavefront phase aberration before correction and $\mathrm{RMS}_{R}$ the value of the residual wavefront phase aberration after correction. The smaller $\mathrm{E}_{f e}$ is, the more precise a wavefront aberration the DM offers. The fitting capabilities of the DM for each Zernike order are shown in Fig. 9.

From Fig. 9 it is obvious that the DM has good fitting capability for the first 7 orders of wavefront aberrations. Generally, lower-order aberrations have the most important effect on intensity distribution and beam quality; therefore,

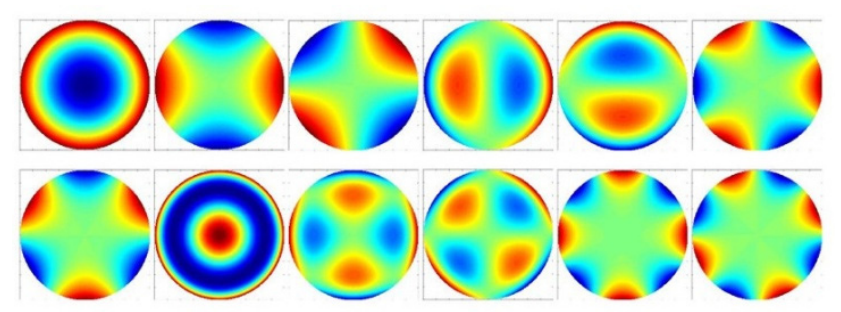

(a)

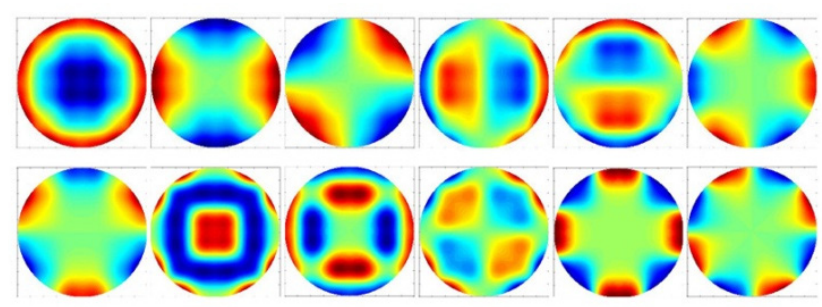

(b)

FIG. 8. Set of aberration modes: (a) 12 Zernike modes (3-14), (b) correcting wavefront (3-14).

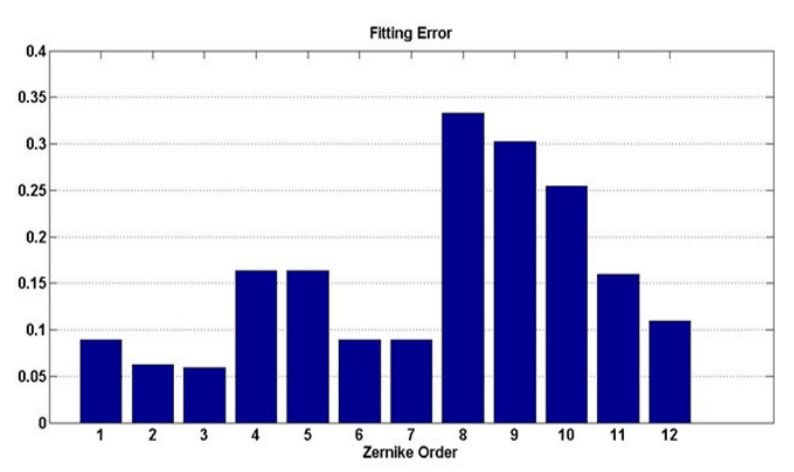

FIG. 9. Fitting capabilities of the DM for each Zernike order. 
we take only the first 7 orders into account. Note that a DM with higher spatial resolution and more actuators can accurately match more types of aberration modes. In our simulations, we introduce an incident wavefront with lower coupling efficiency (shown in Fig. 10).

Our main target is to find the best solution group $\left(u_{1}{ }^{*}\right.$, $\left.u_{2}{ }^{*}\right)$ to fit the Zernike coefficients $\left(v_{1}, v_{2}\right)$ with the MBTS algorithm. We select the initial solutions as $(0,0)$, and the simulation results are shown in Fig. 11. From Fig. 11(a) we know that the searching path is an uneven curve that converges to the final point, namely the best $\operatorname{solution}\left(u_{1}{ }^{*}\right.$, $\left.u_{2}{ }^{*}\right)$. We also find that the final solution group $\left(u_{1}{ }^{*}, u_{2}{ }^{*}\right)$ is $(1.25,0.75)$ from Fig. 11(c) and (d), that the RMS of the residual wavefront phase is about 0.2232 from Fig. 11(b),

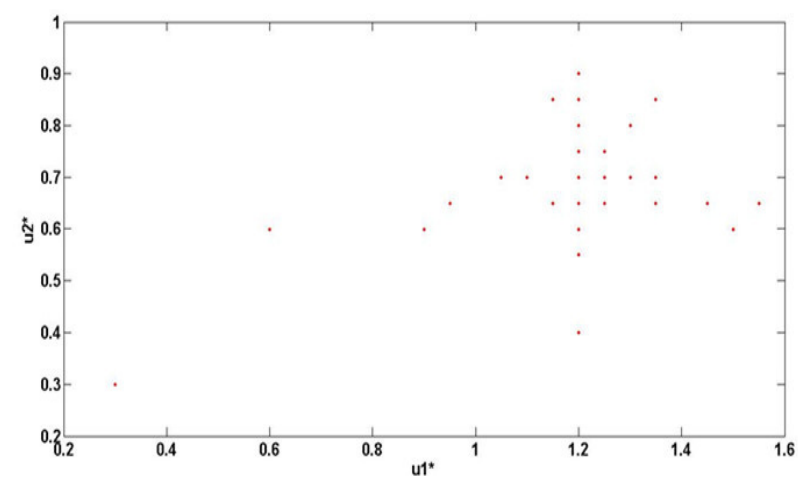

(a)

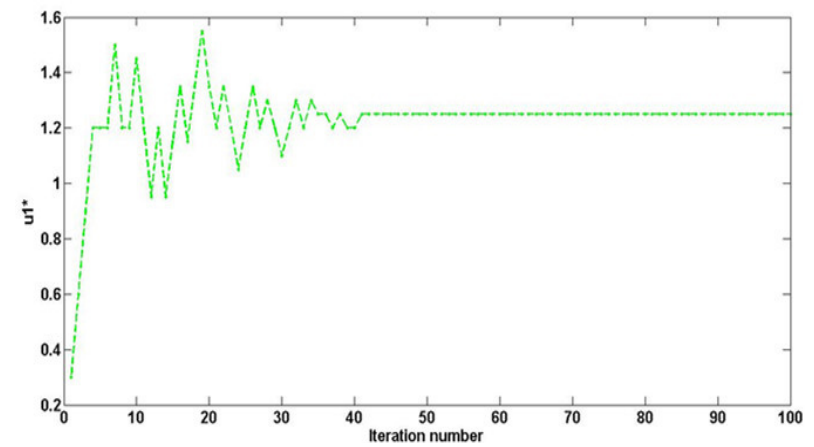

(c)

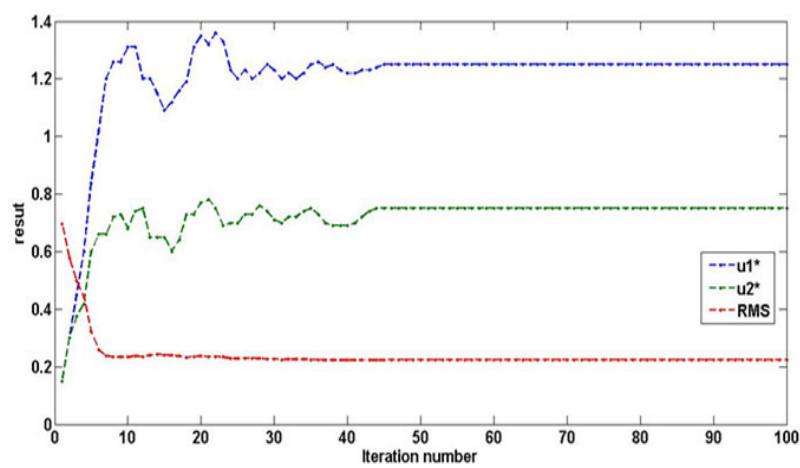

(e)

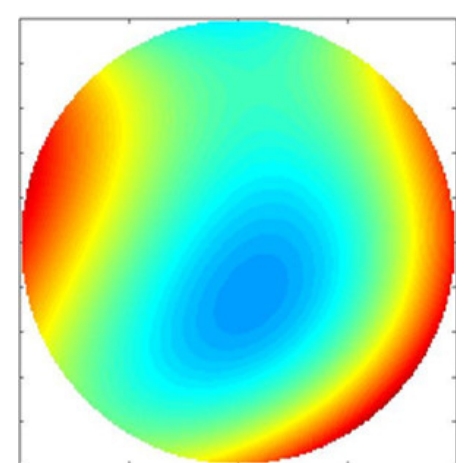

FIG. 10. Introduced wavefront aberration: RMS 0.8484, coupling efficiency $42.81 \%$.

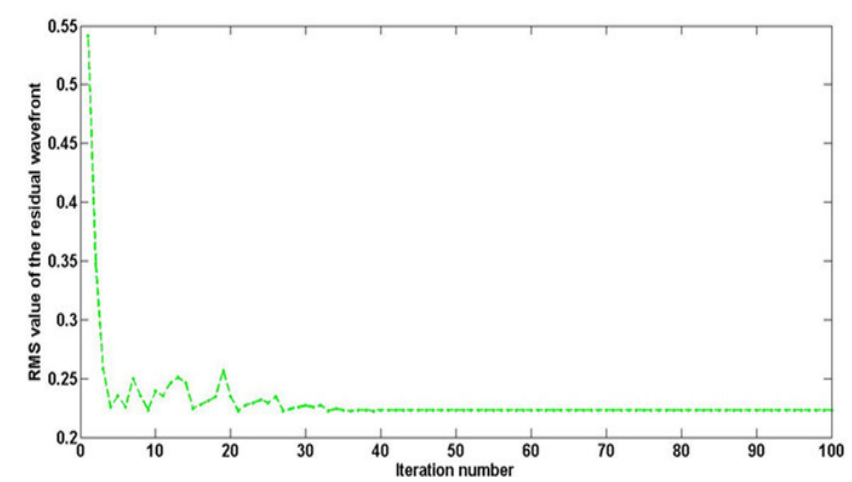

(b)

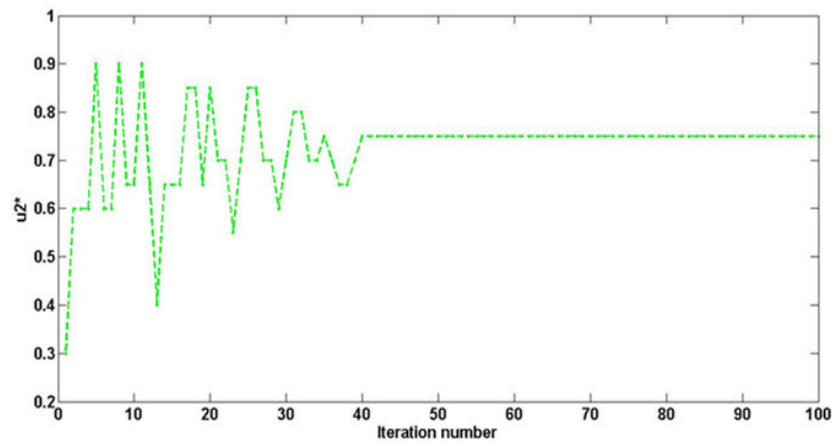

(d)

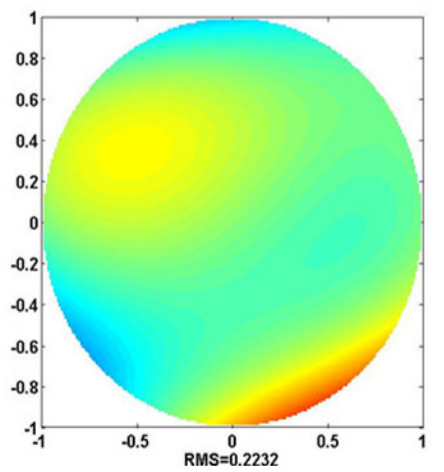

(f)

FIG. 11. Compensation by MBTS algorithm: (a) all points explored during searching, (b) RMS values of residual wavefront for different iterations, (c) solutions of $u_{1}{ }^{*}$ for different iterations, (d) solutions of $u_{2}{ }^{*}$ for different iterations, (e) mean values of current tabu list, (f) residual wavefront after compensation. 


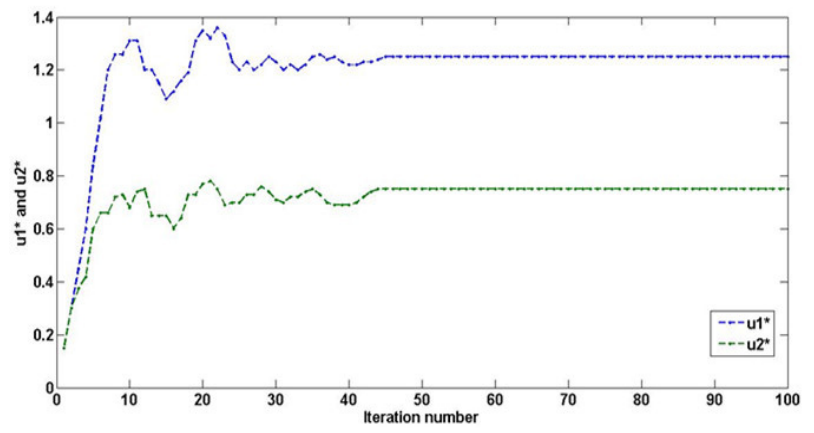

(a)

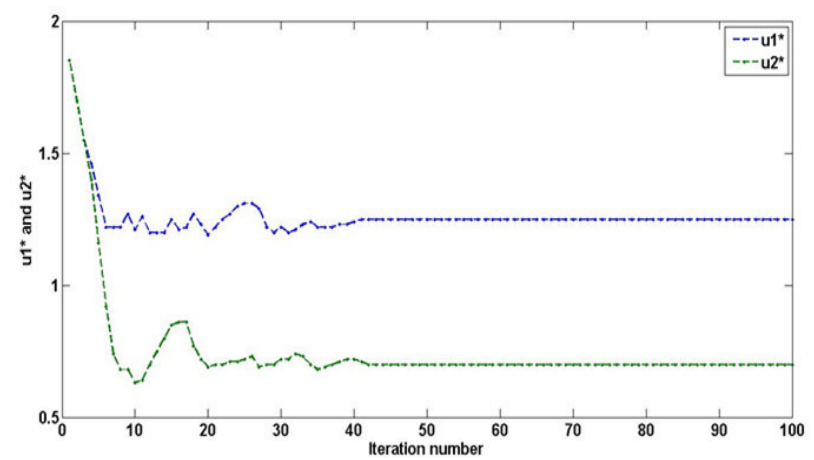

(c)

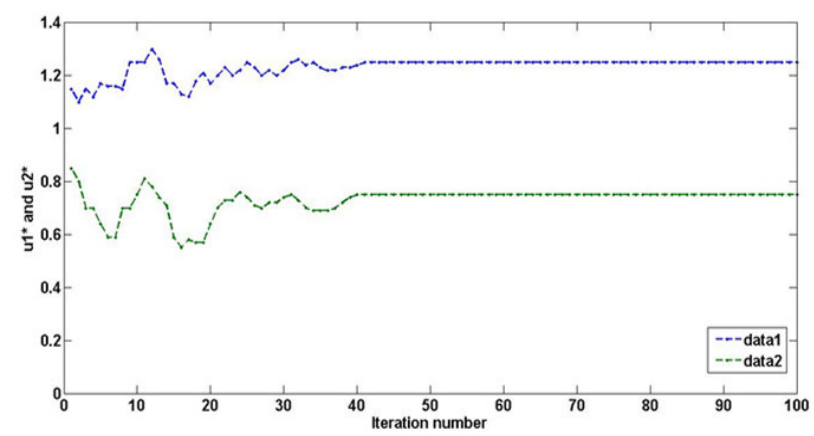

(b)

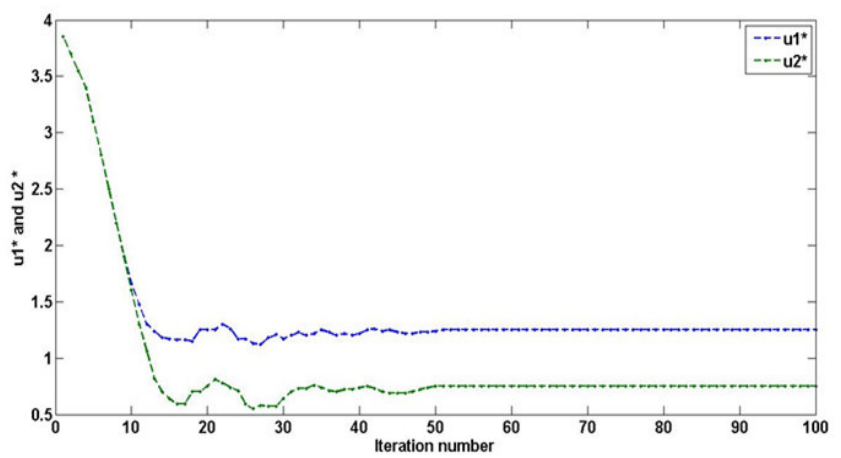

(d)

FIG. 12. Comparison of different initial solutions: initial solution (a) $(0,0),(b)(1,1),(c)(2,2)$, and (d) $(4,4)$.

and that the corresponding coupling efficiency is about $80 \%$, which is a significant improvement. In addition, the number of iterations for this search is about 35 to 40 . However, we can use another method to compute the best solution $\left(u_{1}{ }^{*}, u_{2}{ }^{*}\right)$. The tabu list can record the latest local optimuma selected by the MBTS process. We also can calculate the mean values of the records in the current tabu list during each iteration; the result can be considered as the current best solution in the latest iteration. The results are shown in Fig. 10(e). Note that there are no improvements in finding a better final result, except that the curves are smoother. The residual wavefront phase is shown in Fig. 11(f).

In Fig. 12, we compare the effects on this search process of starting with the different initial solutions $(0,0),(1,1)$, $(2,2)$, and $(4,4)$ respectively. From Fig. 12 we find that different initial solutions lead to different search rates: The initial solution $(4,4)$ offers a faster search process (about 30 iterations), $(2,2)$ takes second place (35 iterations), and $(1,1)$ and $(0,0)$ do less well (about 40 iterations). The choice should be based on some practical experience and several tests.

We also perform a fitting simulation of the remaining Zernike coefficients from $v_{3}$ to $v_{7}$. The result is shown in Fig. 13. Here the initial solution is $(4,4)$, and the final solutions from Fig. 13 are $u_{3}{ }^{*}=-0.5, u_{4}{ }^{*}=0.35, u_{5}{ }^{*}=-0.35$, $u_{6}{ }^{*}=-0.3$, and $u_{7}{ }^{*}=0.3$. The final residual wavefront aberration phase is shown in Fig. 14 with the corresponding RMS value of 0.1379 . We can see that the coupling efficiency is

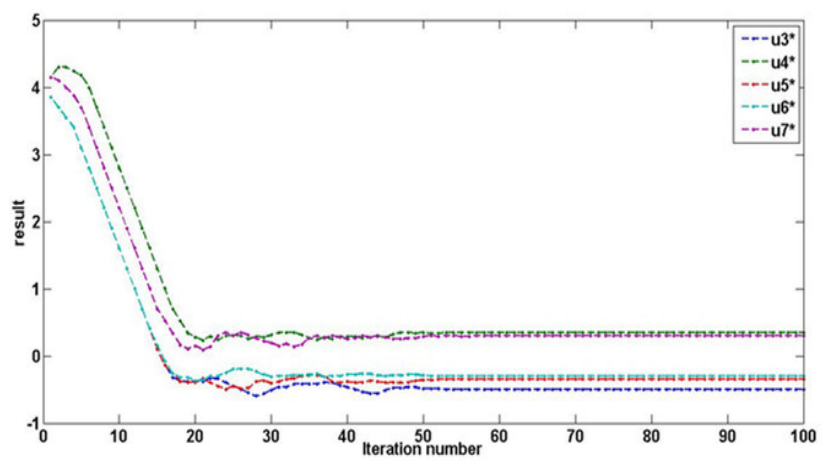

FIG. 13. Solutions for $u_{3}{ }^{*}-u_{7}{ }^{*}$.

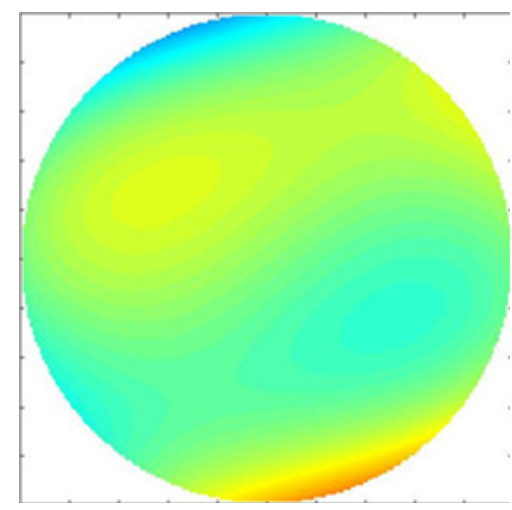

FIG. 14. Final residual wavefront aberration phase. 
improved significantly, from $42.81 \%$ to about $99 \%$.

The variation of coupling efficiency with iteration number is shown in Fig. 15. We select the four different initial solutions mentioned above and find that the final coupling efficiency reaches about $99 \%$ in about 30 iterations.

We also perform some experiments to test the SPGD algorithm; the results before and after correction are shown in Figs. 16 and 17 [21]. In these experiments, we know that the MBTS algorithm is much faster than the conventional SPGD algorithm, usually requiring hundreds of iterations (Fig. 16), while the final coupling efficiency for both algorithms is similar. But the iteration number is largely inferior to that for MBTS even in theory, since the high-dimensional search

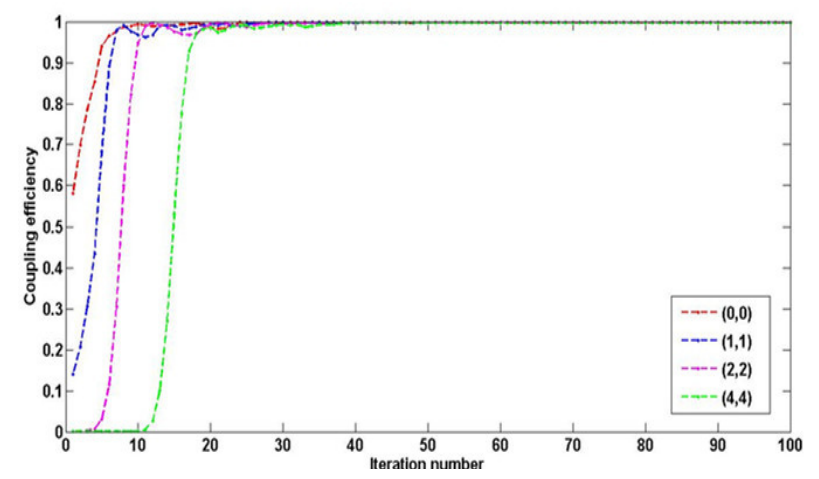

FIG. 15. Coupling efficiency based on MBTS.

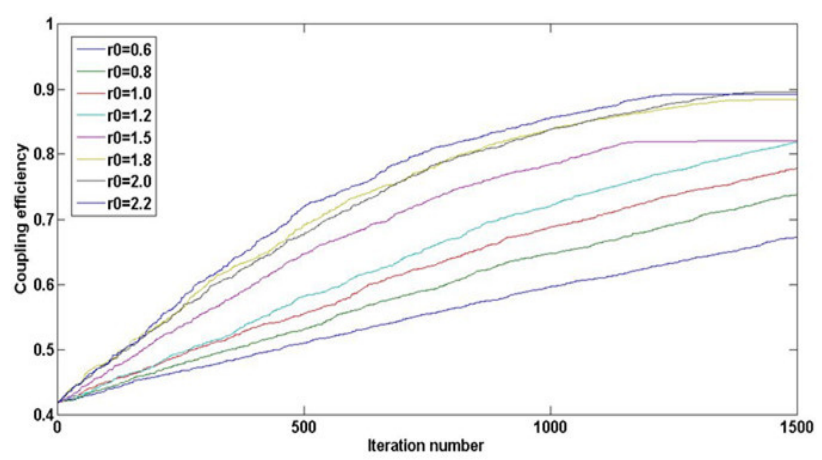

FIG. 16. Coupling efficiency based on SPGD.

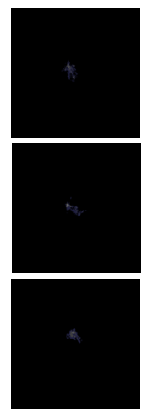

(a)

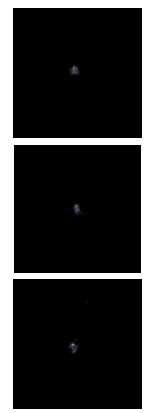

(b)
FIG. 17. Images captured by CCD (a) before and (b) after correction.
TABLE 1. Experimental results using SPGD

\begin{tabular}{c|c}
\hline \hline $\begin{array}{c}\text { Coupling efficiency before } \\
\text { correction }\end{array}$ & $\begin{array}{c}\text { Coupling efficiency after } \\
\text { correction }\end{array}$ \\
\hline $4.35 \%$ & $28.32 \%$ \\
\hline $9.23 \%$ & $31.20 \%$ \\
\hline $13.91 \%$ & $35.96 \%$ \\
\hline
\end{tabular}

space of SPGD reduces the searching rate, while in the MBTS algorithm we project a space with high dimension to a space with lower dimension and decouple them using a new system architecture. Thus the number of iterations will be sharply reduced, but only if the initial point is appropriately selected.

In Fig. 17, from the images received by $\mathrm{CCD}$ we see that the initial coupling efficiency before correction is very low, so that light scintillation is serious, but after correction the coupling efficiency is greatly improved. The corresponding results are in Table 1, and are inferior to the simulation results mainly because of the poor stability of the DM. This means that the DM should generate the identical corrected phase as by the same voltage applied to the actuators, in theory, but in practice it is limited by the skillful processing.

We know that the singleshot correction assumption is a concern for the algorithm convergence speed and atmospheric coherenttime. The SPGD is a typical example in [13], with about 500 iterations in experiment, corresponding to 50 ms. The operation rate is limited by the MEMs DM (7-8 $\mathrm{kHz}$ ) to satisfy the requirements of the $\mathrm{AO}$ system correction. Therefore, the corresponding convergence time of the MBTS is $5 \mathrm{~ms}$ to $6.25 \mathrm{~ms}$ with the new system model, but with some other algorithms (SPGD being the typical one), the time is tens of milliseconds. In the simulation, the time spent by the SPGD is about $90 \mathrm{~ms}$ when the coupling efficiency reaches 0.8 ; in our experiment, it takes 50-60 ms.

It is obvious that the SPGD works far less nicely than the MBTS. However, such a fast search (about 40 iterations) with the MBTS algorithm is at the cost of the construction of complicated equipment, with many sensorless $\mathrm{AO}$ subsystems working simultaneously. It places stringent demands on the experiment, and requires synchronization. This is the first such idea and system proposed in this research area, that many wavefront correctors may work in parallel to achieve better performance.

\section{CONCLUSION}

In this study we propose a novel algorithm, MBTS, to compensate the wavefront aberration from atmospheric turbulence in an FSO communication system based on a new parallel correcting system architecture. The new idea of the proposed 
algorithm and its results in an FSO system are described in detail. Simulations indicate that our proposed MBTS can offer a faster search process during optimization (dozens of iterations) and better final coupling efficiency, compared to the SPGD algorithm. The architecture of a novel and interesting parallel correcting system with multiple adaptive optical (AO) subsystems is also given.

\section{ACKNOWLEDGMENT}

This work is supported by the National Natural Science Foundation of China (No.61171079).

\section{REFERENCES}

1. M. Abtahi, "Suppression of turbulence-induced scintillation in free-space optical communication systems using saturated optical amplifiers," J. Lightwave Technol. 24, 4966-4973 (2007).

2. W. Liu and W. X. Shi, "Free-space optical communication performance analysis with focal plane based wavefront measurement," Opt. Commun. 309, 212-220 (2013).

3. D. Y. Song, H. Y. Such, and C. J. Woo, " $4 \times 10 \mathrm{~Gb} / \mathrm{s}$ terrestrial optical free-space transmission over $1.2 \mathrm{~km}$ using an EDFA preamplifier with $100 \mathrm{GHz}$ channel spacing," Opt. Express 7, 280-284 (2000).

4. D. P. Looze, "Architecture of LQG controllers based on a hybrid adaptive optics system model," European Journal of Control 3, 237-248 (2001).

5. L. Poyneer and J. P. Véran, "Predictive wavefront control for adaptive optics with arbitrary control loop delays," JOSA A 25, 1486-1496 (2008).

6. C. Liu and L. F. Hu, "Modal prediction of atmospheric turbulence wavefront for open-loop liquid-crystal adaptive optics system with recursive least-squares algorithm," Opt. Commun. 285, 238-244 (2012).

7. J. Schwarz and M. Ramsey, "Low order adaptive optics on Z-Beamlet using a single actuator deformable mirror," Opt. Commun. 264, 203-212 (2006).

8. E. Fedrigo, R. Muradore, and D. Zilio, "High performance adaptive optics system with fine tip/tilt control," Control Engineering Practice 17, 122-135 (2009).
9. M. A. Vorontsov, "Decoupled stochastic parallel gradient descent optimization for adaptive optics: Integrated approach for wavefront sensor information fusion," JOSA A $\mathbf{1 9}$, 356-368 (2002).

10. G. L. Liu and H. F. Yang, "Experimental verification of combinational deformable mirror for phase correction," Chinese Optics Letters 5, 559-562 (2007).

11. J. Mooseok, S. Anne, and C. H. Yang, "Optical phase conjugation (OPC)-assisted isotropic focusing," Opt. Express 21, 8781-8792 (2013).

12. M. Monir, A. J. Lowery, and L. B. Du, "Improving performance of optical phase conjugation by splitting the nonlinear element," Opt. Express 21, $4567-4577$ (2013).

13. T. Weyrauch and M. A. Vorontsov, "Atmospheric compensation with a speckle beacon in strong scintillation conditions: Directed energy and laser communication applications," Appl. Opt. 44, 6388-6401 (2005).

14. A. Manunza, M. Marchesi, and F. Pilo, "Tabu Search metaheuristics for global optimization of electromagnetic problems," IEEE Transactions on Magnetics 34, 2690-2693 (1998).

15. H. Song, "Model-based control in adaptive optics systems," Ph. D. Thesis, TU Delft, 5 (2011).

16. L. H. Huang and C. H. Rao, "Wavefront sensorless adaptive optics: A general model-based approach," Opt. Express 19, 371-379 (2001).

17. T. Weyrauch, M. A. Vorontsov, J. W. Gowens, and T. G. Bifano, "Fiber coupling with adaptive optics for free-space optical communication," Free-Space Laser Communication and Laser Imaging 4489, 177-184 (2002).

18. Z. K. Li, J. T. Cao, X. H. Zhao, and W. Liu, "Combinationaldeformable-mirror adaptive optics system for atmospheric compensation in free-space communication," Opt. Commun. 320, 162-168 (2014).

19. Y. M. Sabry, B. Saadany, D. Khalil, and T. Bourouina, "Silicon micromirrors with three-dimensional curvature enabling lensless efficient coupling of free-space light," Light: Science \& Applications 2, e94 (2013).

20. Y. H. Chen, L. Huang, L. Gan, and Z. Y. Li, "Wavefront shaping of infrared light through a subwavelength hole," Light: Science \& Applications 1, e26 (2012).

21. J. T. Cao, X, H, Zhao, Z, K, Li, and W. Liu, "Stochastic parallel gradient descent laser beam control algorithm for atmospheric compensation in free-space optical communication," Optik - International Journal for Light and Electron Optics 125, 6142-6147 (2015). 